\title{
Perioperative Hyperglycemia as a Risk Factor for Early Surgical-Site Infection in Stable Trauma Patients in Orthopedic Unit
}

\author{
Mohd Darulnizam bin Othman, Ahmad Fadzli Sulong \\ Department of Orthopaedic, Traumatology and Rehabilitation, Kulliyyah of \\ Medicine, International Islamic University Malaysia
}

Presenter: Mohd Darulnizam bin Othman

Introduction: Perioperative hyperglycemia resulted from known diabetes mellitus, occult diabetes or stressed-induced hyperglycemia. It is associated with increasing risk of surgical-site infection (SSI). This research is to evaluate relationship between perioperative hyperglycemia and early SSI in stable trauma patients with orthopaedic injuries. Materials and method: We conducted an observational cohort study in Hospital Sultanah Nur Zahirah, Kuala Terengganu over 15 months. We included patients 18 years and older with operative orthopaedic injuries and excluded those with steroid use, multisystem injuries, critically ill or unstable and patients with perioperative infection. Demographics, medical comorbidities, body mass index, type of fracture, HbA1c level in diabetic patients and length of hospital stay were recorded. Fingerprick glucose values were obtained twice daily. Hyperglycemic index $(\mathrm{HGI})$, an area under the glucose curve above the normal range divided by the length of stay, were calculated. Perioperative hyperglycemia was defined by $\mathrm{HGI}$ greater than 1.76. SSI was defined by gross purulence or positive intraoperative culture at reoperation within 30 days. The categorical data were analysed using chi square test while continuous data were analysed using $\mathrm{T}$-test. Results: Total of 90 patients, 15-diabetic and 75 non-diabetic. 10 diabetic and 1 non-diabetic patients were hyperglycemic $(p=0.001)$. Perioperative hyperglycemia was associated with mean age 54.3 year old, having 1 or more co-morbidity or mean admission glucose $13.1 \mathrm{mmol} / \mathrm{L}(\mathrm{p}=0.001)$. There were 12 early SSI giving infection rate $13.3 \%$. Early SSI was associated with HGI 1.76 and more $(p=0.001)$, patients with 1 or more co-morbidity $(p=0.006)$, mean admission glucose of $9.63 \mathrm{mmol} / \mathrm{L}$ $(p=0.001)$. Conclusion: Our study showed perioperative hyperglycemia in stable trauma patients with orthopaedic injuries were associated with SSI. More patients are needed to find association of other variables and more studies are required in determining the acceptable glucose level and optimal treatment of perioperative hyperglycemia in stable trauma patients. 